\title{
Genomic Medicine and Endocrine Autoimmunity as Key to Mitochondrial Disease
}

\author{
Ian James Martins 1,2,3* $^{\text {Iam }}$ \\ ${ }^{1}$ Centre of Excellence in Alzheimer's Disease Research and Care, Sarich Neuroscience Research Institute, Edith Cowan University, Verdun Street, Nedlands, \\ 6009, Western Australia, Australia
}

${ }^{2}$ School of Psychiatry and Clinical Neurosciences, the University of Western Australia, Australia

${ }^{3}$ McCusker Alzheimer's Research Foundation, Hollywood Medical Centre, Australia

*Corresponding author: Ian Martins, School of Medical Sciences, Edith Cowan University, Western Australia 6009, Australia

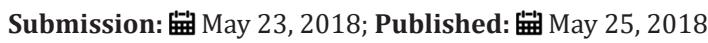

Keywords: Global; Type 3 diabetes; Pandemic; Suprachiasmatic nucleus; Endocrine autoimmunity; Mitophagy; Sirtuin 1; Major histocompatibility

complex; Immunogenic; Genomic medicine; Immune regulation; NAFLD; Species

\section{Editorial}

The diabetes epidemic and the induction of various chronic diseases are expected to affect approx. 592 people by the year 2035. Diabetes and its connections to endocrine autoimmunity [1-3] has become important to metabolic disease with relevance to the non alcoholic fatty liver disease (NAFLD) epidemic and neurodegenerative diseases [4]. The urgency to prevent the largest diabetes epidemic in history has now assessed multiple risk factors

involved with induction of Type 3 diabetes connected to various chronic diseases [4]. Interest in Type 3 diabetes has accelerated in the past 15 years with the critical importance of anti-aging genes [5,6] with relevance to autoimmune disease and mitophagy [7] and the global diabetes epidemic. The regulation of these genes is controlled by the heat shock gene Sirtuin 1 (Sirt 1 ) that is connected to appetite, immune and core body temperature regulation with relevance tomitophagy in NAFLD and metabolic disease.

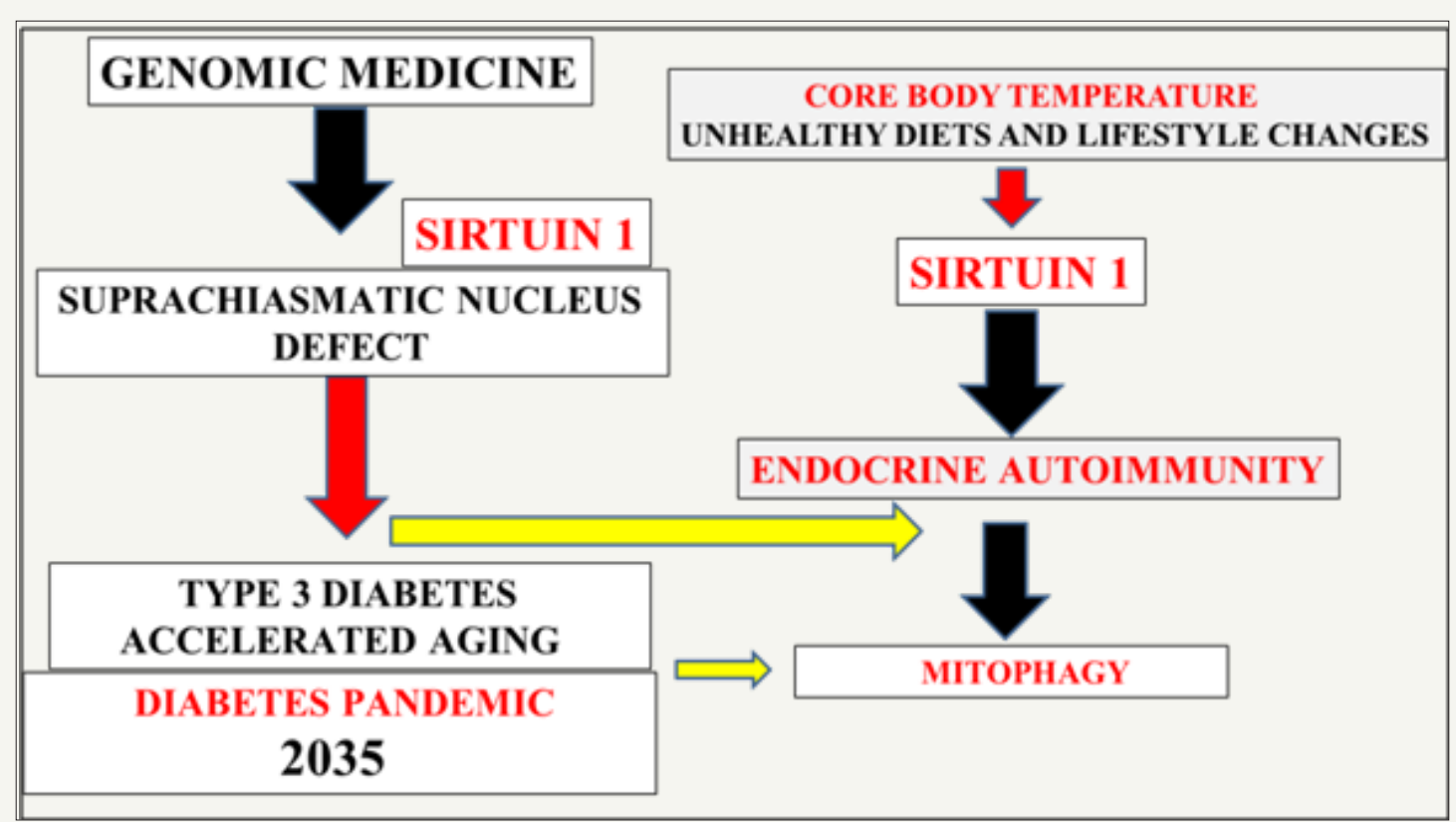

Figure 1: Genomic medicine and regulation of the heat shock gene Sirtuin 1 (Sirt 1) is connected to Type 3 diabetes, endocrine autoimmunity and mitochondrial disease. Unhealthy diets and lifestyle changes determine defective suprachiasmatic nucleus regulation (SCN) relevant to Type 3 diabetes/endocrine autoimmunity and the predicted diabetes pandemic by the year 2035 . 
Diabetes as an endocrine disease now indicates stress [8] as a major factor in the induction of brain aging and Type 3 diabetes connected to endocrine autoimmunity [9]. The increased global susceptibility to insulin resistance associated with brain aging and neurodegenerative diseases now indicate neuron vulnerability to mitophagyis critical to the reversal of the diabetes pandemic. Interests in Sirt 1 in Type 3 diabetes with relevance to insulin resistance and autoimmune disease has accelerated to prevent accelerated mitochondrial apoptosis (Figure 1) and brain aging [7]. Sirt 1 is a nicotinamide adenine dinucleotide (NAD +) dependent class III histone deacetylase (HDAC) and as a heat shock gene $[5,10]$ is involved in the deacetylation of heat shock factor 1 (HSF 1), regulation of heat shock proteins (HSP) and nitric oxide metabolism connected to natural killer cell activity, mitophagy and autoimmune disease in neuro degeneration and various chronic diseases.

Type 3 diabetes and endocrine autoimmunity now involves attack by the immune system and implicates the major histocompatibility complex (MHC) to be relevant to diabetes and endocrine autoimmunity.MHC molecules are cell-surface glycoproteins that regulate adaptive immune responses and interference with MHC gene expression at the level of transcription is involved with autoimmune disease [11,12]. Recent advances in genetics now reveal Sirt 1 to be involved with immune and endocrine disturbances (Figure 1) in these diseases [7,10]. Sirt 1 as a deacetylase targets transcription factors to adapt gene expression to immune regulation, metabolic activity and insulin resistance. Sirt 1 regulates the major histocompatibility complex class II (MHCII) genes at the level of transcription important to the immune recognition and autoimmune disease in various species [13-16]. Sirt 1 deacetylates the master regulator CIITA (adaptive immune response) that determines the expression ofMHC-II genes and autoimmune disease [14, 16-18].

The connections between diabetes, endocrine dysfunction and mitochondrial disease involve Sirt 1 and various hormones involved with endocrine autoimmunity and mitochondrial disease [19-22]. Sirt 1 is involved in mitochondrial biogenesis [23] and its regulation of the suprachiasmatic nucleus (SCN) is connected to various brain hormones and immunological diseases [7]. Sirt 1 regulation of hormones such as apelin, brain derived neurotrophic factor, growth hormone, neuropeptide $Y$, adiponectin and fibroblast growth factor 21 are connected to prevention of autoimmune disease [7,24-26]. The accelerated brain aging in the current global diabetes pandemic may now be relevant to endocrine autoimmune disease, mitophagy and cancer [19-22]. Endocrine treatment of mitochondrial disease [27-32] may be completely inhibited by Sirt1transcriptional dysregulation and relevant to mitochondrial HSP-antigen presentation [33-35].

Genomic medicine and maintenance of the SCN is critical for prevention of endocrine autoimmunity with relevance to mitochondrial disease (Figure 1). Core body temperature [36], unhealthy diets [37] and lifestyle changes/stress [8] in diabetes can inactivate the SCN with accelerated Type 3 diabetes and neuro degeneration. The connections between endocrinology and autoimmune disease are determined by Sirt 1 repression of MHC genes that may involve the induction of antigenic and immunogenic proteins [38] and lipids [7] with relevance to mitochondrial apoptosis. Specific diets with Sirt 1 activators/Sirt 1 inhibitors $[4,38]$ in the developing world are required to reverse Sirt 1 repression and maintain immune recognition essential for human survival and prevention of diabetes and various chronic diseases. Sirt 1 as the anti-aging gene now determines longevity [39] in various species with its repression connected to the induction of endocrine autoimmunity and hyperglycemic mitochondrial apoptosis in the global diabetes epidemic $[2,3]$.

\section{Conclusion}

Genomic medicine treatment of diabetics is critical in the current global diabetes epidemic to prevent the expected diabetes pandemic predicted to occur by the year 2035 . The major concern with Type 3 diabetes in the global population is related to a defective SCN with relevance to uncontrolled peripheral glucose levels and endocrine autoimmune disease. Appetite regulation and genomic medicine are critical to Sirt 1's regulation of the MHC genes with relevance to maintenance of immune recognition and endocrine hormone treatment of mitophagy. In the developing world the major concern for a diabetes pandemic is mitophagy and will require diets with Sirt 1 activators to prevent Type 3 diabetes, endocrine autoimmunity and mitochondrial disease.

\section{Acknowledgements}

This work was supported by grants from Edith Cowan University, the McCusker Alzheimer's Research Foundation and the National Health and Medical Research Council.

\section{References}

1. Michels AW, Eisenbarth GS (2010) Immunologic endocrine disorders. J Allergy ClinImmunol 125(2 Suppl 2): S226-S237.

2. Zimmet PZ (2017) Diabetes and its drivers: the largest epidemic in human history? Clin Diabetes Endocrinol 3: 1.

3. Zimmet PZ, Alberti KG (2016) Epidemiology of diabetes-status of a pandemic and issues around metabolic surgery. Diabetes Care 39(6): 878-883.

4. Martins IJ (2017) Nutrition therapy regulates caffeine metabolism with relevance to NAFLD and induction of type 3 Diabetes. J Diabetes Metab Disord 4: 019.

5. Martins IJ (2016) Type 3 diabetes with links to NAFLD and other chronic diseases in the western world. Int J Diab Metab Disordr 1: 1-5.

6. Martins IJ (2016) Diet and nutrition reverse Type 3 diabetes and accelerated aging linked to global chronic diseases. J Diab Res Ther 2(2): 1-6.

7. Martins IJ (2018) Appetite control and biotherapy in the management of autoimmune induced global chronic diseases. J ClinImmunol Res 2(1): $1-4$.

8. Martins IJ (2015) Nutritional diets accelerate amyloid beta metabolism and prevent the induction of chronic diseases and Alzheimer's disease. Scientific Research 5(1): 1-48.

9. Anderson MS (2008) Update in endocrine autoimmunity. J Clin Endocrinol Metab 93(10): 3663-3670. 
10. Martins II (2017) Single gene inactivation with implications to diabetes and multiple organ dysfunction syndrome. J Clin Epigenet 3(3): 1-8.

11. Sommer S (2005) The importance of immune gene variability (MHC) in evolutionary ecology and conservation. Front Zool 2: 16.

12. Choi NM, Majumder P, Boss JM (2011) Regulation of major histocompatibility complex class II genes. Curr Opin Immunol 23(1): 81-87.

13. Fernando MMA, Stevens CR, Walsh EC, Jager PI, Goyette P, et al. (2008) Defining the role of the MHC in autoimmunity: A review and pooled analysis. PLoS Genet 4(4): e1000024.

14. Martínez A, Lopez MS, Varadé J, Mas A, Martín MC, et al. (2007) Role of the MHC2TA gene in autoimmune diseases. Ann Rheum Dis 66(3): 325329.

15. Dema B, Martínez A, Arquero MF, Maluenda C, Polanco I, et al. (2009) Autoimmune disease association signals in CIITA and KIAA0350 are not involved in celiac disease susceptibility. Tissue Antigens 73(4): 326-329.

16. Waldburger JM, Palmer G, Seemayer C, Lamacchia C, Finckh A, et al. (2011) Autoimmunity and inflammation are independent of class II transactivator type PIV-dependent class II major histocompatibility complex expression in peripheral tissues during collagen-induced arthritis. Arthritis Rheum 63(11): 3354-3363.

17. Wu X, Kong X, Chen D, Li H, Zhao Y, et al. (2011) SIRT1 links CIITA deacetylation to MHC II activation. Nucleic Acids Res 39(22): 9549-9558.

18. Masternak K, Mottet AM, Villard J, Zufferey M, Steimle V, et al. (2000) CIITA is a transcriptional coactivator that is recruited to MHC class II promoters by multiple synergistic interactions with an enhanceosome complex. Genes Dev 14(9): 1156-1166.

19. Gadi IS, Haas RH, Falk MJ, Goldstein A, McCormack SE (2018) Endocrine disorders in primary mitochondrial disease. J Endocr Soc 2(4):361-373.

20. Roden M (2013) Mitochondrial endocrinology--mitochondria as key to hormones and metabolism. Mol Cell Endocrinol 379(1-2): 1

21. Chow J, Rahman J, Achermann JC, Dattani MT, Rahman S (2017) Mitochondrial disease and endocrine dysfunction. Nat Rev Endocrinol 13(2): 92-104.

22. Schaefer AM, Walker, Turnbull DM, Taylor RW (2013) Endocrine disorders in mitochondrial disease. Mol Cell Endocrinol 379(1-2): 2-11.

23. Tang B (2016) Sirt1 and the mitochondria. Mol Cells 39(2): 87-95.

24. Martins IJ (2016) Anti-aging genes improve appetite regulation and reverse cell senescence and apoptosis in global populations. AAR 5(1): $9-26$

25. Martins IJ (2016) The role of clinical proteomics, lipidomics, and genomics in the diagnosis of alzheimer's disease. Proteomes 4(2) 1-19.
26. Yamamoto M, Iguchi G, Fukuoka H, Suda K, Bando H, et al. (2013) SIRT1 regulates adaptive response of the growth hormone--insulin-like growth factor-I axis under fasting conditions in liver. Proc Natl Acad Sci USA 110(37): 14948-14953.

27. Iwabu M, Yamauchi T, Iwabu MO, Sato K, Nakagawa T, et al. (2010) Adiponectin and AdipoR1 regulate PGC-1 $\alpha$ and mitochondria by $\mathrm{Ca} 2+$ and AMPK/SIRT1. Nature 464(7293): 1313-1319.

28. Pisarenko O, Shulzhenko V, Studneva I, Pelogeykina Y, Timoshin A, et al. (2015) Structural apelin analogues: mitochondrial ROS inhibition and cardiometabolic protection in myocardial ischaemia reperfusion injury. Br J Pharmacol 172(12): 2933-2945.

29. Su B, Ji YS, Sun XL, Liu XH, Chen ZY (2014) Brain-derived neurotrophic factor (BDNF)-induced mitochondrial motility arrest and presynaptic docking contribute to BDNF-enhanced synaptic transmission. J Biol Chem 289(3): 1213-1226.

30. Su B, Ji YS, Sun XH, Liu XH, Chen ZY (2014) Brain-derived neurotrophic factor controls mitochondrial transport in neurons. J Biol Chem 289(3): 1213-1226.

31. Quintos JB, Hodax JK, Ellis BAG, Phornphutkul C, Wajnrajch MP, et al. (2017) Response to growth hormone deficiency in mitochondrial disorders. J Pediatr Endocrinol Metab 30(4): 483-484

32. Davis RL, Liang C, Hildebrand FE, Riley C, Needham M, et al. (2013) Fibroblast growth factor 21 is a sensitive biomarker of mitochondrial disease. Neurology 81(21): 1819-1826.

33. Murshid A, Gong J, Calderwood SK (2012) The role of heat shock proteins in antigen cross presentation. Front Immunol 3: 63.

34. Bonifaz L, Silva MC, Dotor E0, Villegas EL, García FS (2014) A role for mitochondria in antigen processing and presentation. Immunology.

35. Baden P, Deleidi M (2016) Mitochondrial antigen presentation: a vacuolar path to autoimmunity in parkinson's disease. Trends Immunol 37(11): 719-721.

36. Martins IJ (2017) Regulation of core body temperature and the immune system determines species longevity. Curr Updates Gerontol 1: 6.1

37. Martins IJ (2015) Overnutrition determines LPS regulation of mycotoxin induced neurotoxicity in neurodegenerative diseases. Int J Mol Sci 16(12): 29554-29573.

38. Martins IJ (2018) Heat shock gene inactivation and protein aggregation with links to chronic diseases. Diseases 6(39): 1-5.

39. Martins IJ (2018) Anti-aging gene linked to appetite regulation determines longevity in humans and animals. IJOAR 1(6): 1-4
Creative Commons Attribution 4.0 International License

For possible submissions Click Here

\section{Submit Article}

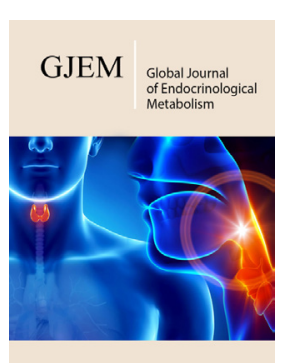

Global Journal of Endocrinological Metabolism

\section{Benefits of Publishing with us}

- High-level peer review and editorial services

- Freely accessible online immediately upon publication

- Authors retain the copyright to their work

- Licensing it under a Creative Commons license

- Visibility through different online platforms 